Departamento de Ciência e Tecnologia, Secretaria de Ciência, Tecnologia e Insumos

Estratégicos, Ministério da Saúde

Correspondência | Correspondence:

Decit - Departamento de Ciência e Tecnologia

do Ministério da Saúde

Esplanada dos Ministérios

Bloco G sala 845

70058-900 Brasília, DF, Brasil

Texto de difusão técnico-científica do

Ministério de Saúde.

\section{9a Reunião Nacional da SBPC: Amazônia - Desafio Nacional em Belém, 2007}

\section{$59^{\text {th }}$ SBPC National Meeting: Amazon - National Challenge in Belém, 2007}

\section{ANTECEDENTES}

Após dois anos de reuniões realizadas na região amazônica dedicadas ao tema "Amazônia - Desafio Nacional de Belém, realizou-se, no período de 8 a 13 de julho de 2007, na cidade de Belém, Pará, a 59ª Reunião Nacional da Sociedade Brasileira para o Progresso da Ciência (SBPC). Duas perguntas nortearam a agenda de atividades da reunião: 1) Existe um Projeto de Nação que inclua a Aazônia?; 2) Seríamos uma Nação sem a Amazônia?

No contexto dessas duas perguntas, em 2003 o Governo Federal iniciou a condução da formulação do Programa de Desenvolvimento Sustentável da Amazônia, por meio dos Ministérios da Integração Nacional e do Meio Ambiente. Esse Programa abrange cinco eixos temáticos prioritários: produção sustentável com tecnologia avançada, novo padrão de financiamento, gestão ambiental e ordenamento territorial, infra-estrutura para o desenvolvimento e inclusão social e cidadania. Dentro da questão da inclusão social e cidadania está inserida a temática da saúde, que vem sendo coordenada pela Secretaria Executiva do Ministério da Saúde.

Uma série de atividades foi desenvolvida visando à construção conjunta de um plano de saúde para a região. Entre elas, em agosto de 2003 em Manaus, Amazonas, realizou-se a $1^{\text {a }}$ Oficina de Planejamento Regional em Saúde: construindo uma agenda de saúde para a Amazônia Legal. O objetivo dessa oficina foi identificar problemas e dificuldades no campo da atenção à saúde, da gestão do trabalho e do financiamento de ciência e tecnologia. Também foram indicadas ações prioritárias e definidas linhas gerais para a elaboração do Plano de Ações Integradas para a Amazônia Legal.

Ainda em Manaus, foram constituídos seis grupos temáticos para discussão de problemas prioritários e formulação de estratégias, dentre os quais o de Ciência, Tecnologia e Inovação em Saúde. Nesse grupo discutiram-se as questões referentes à formação de recursos humanos em Ciência, Tecnologia e Inovação em Saúde; informática e informação em saúde; baixa inovação tecnológica em saúde; necessidade de investimentos na área; e definição de pesquisa em saúde na região.

Em outubro de 2003, em Belém, realizou-se a $1^{\text {a }}$ Oficina de Trabalho de Ciência, Tecnologia e Inovação em Saúde na Região Amazônica. Os objetivos foram aprofundar os resultados da $1^{\text {a }}$ Oficina de Planejamento Regional em Saúde, estabelecer ações prioritárias para a região e definir prioridades de pesquisa em saúde.

Nessa reunião de outubro, foram constituídos os seguintes grupos temáticos: saúde e meio ambiente - grupos em situação de vulnerabilidade e estratégias de desenvolvimento sustentável; recursos humanos - educação e trabalho em saúde; e financiamento de projetos para desenvolvimento de ciência, tecnologia e inovação em saúde \& políticas de assistência farmacêutica. Na ocasião foi assinado o Acordo Multilateral de Cooperação Técnico-Científica das Instituições de Saúde da Amazônia, visando à cooperação entre a Fundação Oswaldo Cruz (Fiocruz) e instituições regionais para o desenvolvimento da área de saúde na região da Amazônia Legal.

Dando continuidade às atividades, em dezembro de 2003, realizou-se a $2^{\mathrm{a}}$ Reunião do Acordo Multilateral de Cooperação Técnico Científica/ $2^{\mathrm{a}}$ Oficina de Trabalho Ciência, Tecnologia e Inovação em Saúde na região Amazônica, na cidade de Porto Velho, Rondônia. Esses eventos tiveram o objetivo de consolidar propostas na área de pesquisa, desenvolvimento e formação de recursos humanos. Para a área de recursos humanos foi proposta a implantação de novos cursos de mestrado e doutorado em Ciências da Saúde, com ênfase para as áreas de saúde coletiva, doenças infecciosas e parasitárias, saúde e ambiente, áreas clínicas e epidemiologia clínica. Foram, ainda, delineados alguns temas prioritários para o desenvolvimento de pesquisa em saúde na Amazônia.

Como resultado de todo esse processo de discussão, foram estabelecidos sete eixos temáticos de pesquisa em saúde prioritários para a região, denominados redes 


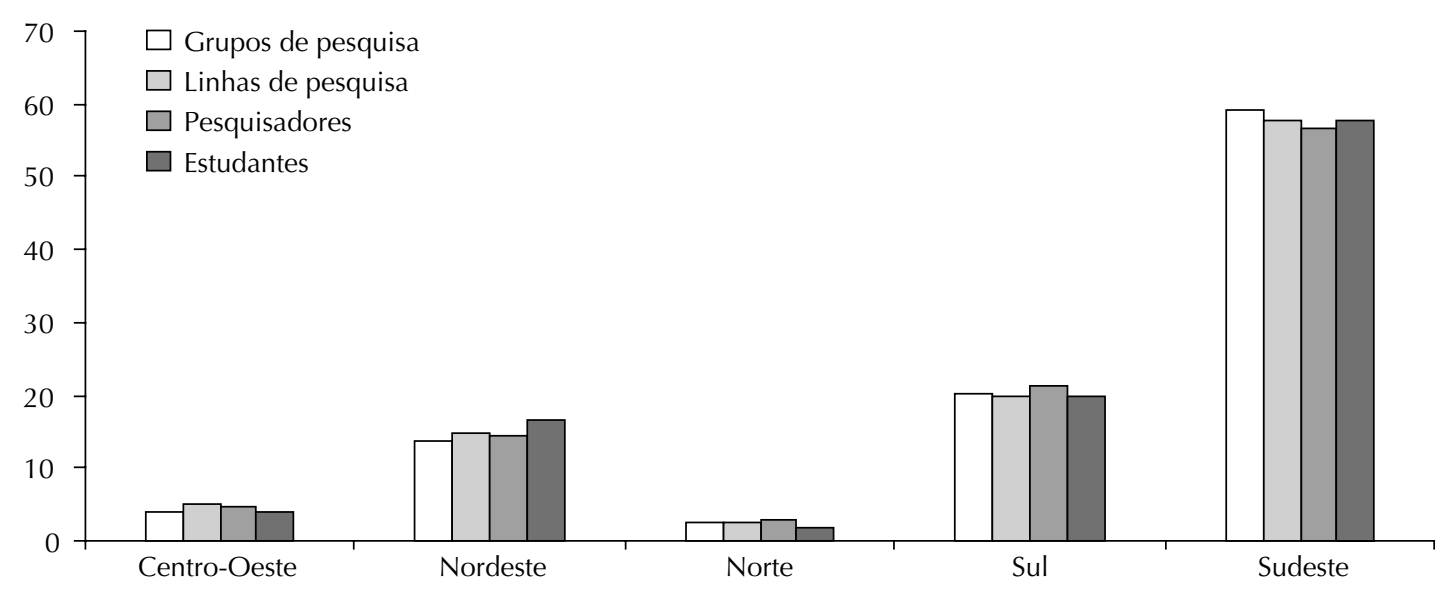

Fonte: Conselho Nacional de Desenvolvimento Científico e Tecnológico. Censo 2004.

Figura. Proporção de grupos, linhas de pesquisa, pesquisadores e estudantes segundo a grande área das Ciências da Saúde e regiões. Brasil, 2004.

de pesquisa, sobre os temas: 1) malária; 2) micobacterioses; 3) síndromes febris ictero-hemorrágicas agudas; 4) avaliação de programas e de serviços em saúde; 5) saúde e ambiente em espaços e em grupos sociais em situação de vulnerabilidade; 6) nutrição e alimentação; e 7) fitoterápicos.

Um oitavo grupo de trabalho foi constituído para discutir a problemática da formação de recursos humanos em saúde.

Com base nos eixos temáticos acima mencionados, o Departamento de Ciência e Tecnologia, da Secretaria de Ciência, Tecnologia e Insumos Estratégicos do Ministério da Saúde (Decit/SCTIE/MS), implementou o Programa Pesquisa para o SUS / Saúde Amazônia. Esse programa contou da publicação de duas chamadas públicas para financiamento de projetos de pesquisa relacionados aos temas prioritários, onde foram apoiadas 29 pesquisas, distribuídas em institutos sediados na região amazônica. Uma outra ação do Decit/SCTIE/MS, específica para a região, envolveu o apoio a pesquisas em saúde para a área de abrangência da BR163.

\section{AMAZÔNIA: DESAFIO NACIONAL}

O Ministério da Saúde promoveu, durante a $59^{\mathrm{a}}$ Reunião, três dias de encontro sobre saúde na região amazônica, com os seguintes temas:

1. O Ministério da Saúde e a redução das desigualdades regionais na Amazônia: desafios e perspectivas:

- gestão do trabalho e educação em saúde: Região Amazônica;
- desafios para ampliação do acesso aos serviços de saúde na região amazônica;

- redução das desigualdades em pesquisa: o exemplo amazônico.

2. Prioridades sanitárias para a região amazônica:

- políticas de vigilância em saúde e doenças emergentes na região amazônica;

- saúde da mulher na região amazônica;

- saúde mental na região amazônica;

- promoção em saúde na região amazônica.

3. Perspectivas de desenvolvimento tecnológico em saúde para a região amazônica:

- saúde e meio ambiente na região amazônica;

- inovação em saúde para a região amazônica;

- biotecnologia em saúde para a região amazônica;

- C\&T na região amazônica: a experiência do Pará.

Durante o primeiro encontro, a SCTIE/Decit abordou o tema "Redução das desigualdades regionais em pesquisa: o exemplo amazônico". Foram apresentadas as ações desenvolvidas pelo Decit com foco específico para a região amazônica e os resultados alcançados. Atualmente existem cerca de 270 ações que contam com o apoio financeiro do Decit,* distribuídas em 43 instituições da região amazônica, entre pesquisas, apoio a Comitês de Ética em Pesquisa, Centros de Pesquisa Clinica e projetos estratégicos. Os outros temas dos

\footnotetext{
* Fonte: Banco de dados interno do Decit.
} 
encontros forma apresentados pela Secretaria de Gestão do Trabalho e Educação em Saúde (SGETS), Secretaria de Atenção à Saúde, Secretaria de Vigilância em Saúde, pela Fiocruz, pelo Instituto Evandro Chagas, pela Financiadora de Estudos e Projetos do Ministério da Ciência e Tecnologia (Finep/MCT), pela Agência Brasileira de Desenvolvimento Industrial (ABDI) e pela Secretaria de Ciência e Tecnologia do Estado do Pará.

Foram abordados ainda os principais indicadores de ciência, tecnologia e inovação em saúde para os estados da região amazônica. Há carência de investimentos para a consolidação de uma base técnico-científica, capaz de participar ativamente do processo de desenvolvimento local. Isso se reflete na distribuição discrepante dos grupos de pesquisa, pesquisadores, estudantes, técnicos e linhas de pesquisa, para todas as áreas do conhecimento. Para a grande área das Ciências da Saúde, as disparidades se repetem, com baixa concentração de recursos humanos na região Norte, que engloba maior parte dos estados da região da Amazônia Legal (Figura).
O II Encontro da Rede Pan-amazônica de Ciência, Tecnologia e Inovação em Saúde também aconteceu durante a $59^{\mathrm{a}}$ Reunião, contou com a participação do Ministério da Saúde, representado pela SCTIE. A Rede Pan-Amazônica (formada pelos governos do Brasil, Bolívia, Colômbia, Equador, Guiana, Peru, Suriname e Venezuela) tem como objetivo responder aos desafios na área da saúde de forma conjunta. Participam, também, da Rede como entidades constituintes, a Fiocruz, a Organização do Tratado de Cooperação Amazônica (OTCA) e a Associação de Universidades Amazônicas (Unamaz). A Rede Pan-amazônica objetiva a criação de uma agenda supranacional na área da saúde, que possibilite entender o significado da saúde para seus povos e a importância da questão no âmbito da globalização. A criação de sinergias no desenvolvimento de Ciência, Tecnologia e Inovação em Saúde (CT\&I/S), bem como a articulação dos atores à pesquisa e qualificação na Amazônia, também são metas a serem atingidas pela Rede Pan-amazônica. 